\title{
Surgical intensive care unit clinician estimates of the adequacy of communication regarding patient prognosis
}

Rebecca A Aslakson ${ }^{1 *}$, Rhonda Wyskiel ${ }^{2}$, Dauryne Shaeffer ${ }^{2}$, Marylou Zyra², Nita Ahuja ${ }^{3}$, Judith E Nelson ${ }^{4}$, Peter J Pronovost ${ }^{1}$

\begin{abstract}
Introduction: Intensive care unit (ICU) patients and family members repeatedly note accurate and timely communication from health care providers to be crucial to high-quality ICU care. Practice guidelines recommend improving communication. However, few data, particularly in surgical ICUs, exist on health care provider opinions regarding whether communication is effective.

Methods: To evaluate ICU clinician perceptions regarding adequacy of communication regarding prognosis, we developed a survey and administered it to a cross section of surgical ICU nurses, surgical ICU physicians, nurse practitioners (NPs), and surgeons.

Results: Surgeons had a high satisfaction with communication regarding prognosis for themselves (90\%), ICU nurses (85\%), and ICU physicians and NPs (85\%). ICU nurses noted high satisfaction with personal (82\%) and ICU physician and NP (71\%) communication, but low (2\%) satisfaction with that provided by surgeons. ICU physicians and NPs noted high satisfaction with personal (74\%) and ICU nurse (88\%) communication, but lower (23\%) satisfaction with that provided by surgeons. ICU nurses were the most likely (75\%) to report speaking to patients and patient families regarding prognosis, followed by surgeons (40\%), and then ICU physicians and NPs (33\%). Surgeons noted many opportunities to speak to ICU nurses and ICU physicians and NPs about patient prognosis and noted that comments were often valued. ICU physicians and NPs and ICU nurses noted many opportunities to speak to each other but fewer opportunities to communicate with surgeons. ICU physicians and NPs thought that their comments were valued by ICU nurses but less valued by surgeons. ICU nurses thought that their comments were less valued by ICU physicians and NPs and surgeons.

Conclusions: ICU nurses, surgeons, and ICU intensivists and NPs varied widely in their satisfaction with communication relating to prognosis. Clinician groups also varied in whether they thought that they had opportunities to communicate prognosis and whether their concerns were valued by other provider groups. These results hint at the nuanced and complicated relationships present in surgical ICUs. Further validation studies and further evaluations of patient and family member perspectives are needed.
\end{abstract}

\section{Introduction}

Intensive care unit (ICU) patients and their family members repeatedly identify communication as essential to high-quality ICU care [1,2]. They emphasize the importance of "timely, clear, and compassionate communication

\footnotetext{
* Correspondence: raslaks1@jhmi.edu

'Department of Anesthesiology and Critical Care Medicine, The Johns Hopkins School of Medicine, 600 North Wolfe Street, Baltimore, MD, 21287, USA

Full list of author information is available at the end of the article
}

by clinicians" [3]. Most want to know their physicians' estimates of prognosis, even if the prognosis is uncertain [4-6]. Patients and families benefit from proactive communication because it decreases the use of unwanted and ineffective treatments in the ICU $[7,8]$, reduces ICU length of stay [9], and promotes earlier consensus around goals of care [10]. Consequently, professional societies and practice guidelines recommend that ICU clinicians communicate proactively with patients and patient families [11-15].
C Biomed Central

() 2010 Aslakson et al.; licensee BioMed Central Ltd. This is an open access article distributed under the terms of the Creative Commons Attribution License (http://creativecommons.org/licenses/by/2.0), which permits unrestricted use, distribution, and reproduction in any medium, provided the original work is properly cited. 
As in other settings, optimal communication in the ICU includes discussion of the disease, prognosis, goals of care, treatment options, and patient preferences. Proactive ICU communication should also address preferences regarding resuscitation. When burdens of intensive care treatment outweigh benefit, discussion of limitation of this treatment is also appropriate. Evidence suggests that clinicians working in surgical ICUs find this type of communication to be particularly challenging. Surgical textbooks contain scant content related to communication of distressing news or goal setting [16]. The "rescue culture" that dominates many surgical ICUs may also further impede such discussions [17-20]. Moreover, as these discussions can reveal varying goals of care between health care providers, they often cause moral distress and conflict [21,22].

Data from our own institution suggest that patients receiving surgical ICU care for more than 7 days have a high (41\%) rate of in-hospital mortality [23]. Thus, the specific aim of this article is to investigate the views and experiences of clinicians working in the surgical ICU regarding the adequacy and efficacy of communication regarding prognosis for surgical ICU patients.

\section{Materials and methods}

In September and October of 2009, we completed a cross-sectional, computer-based survey to assess health care-provider opinions concerning communication regarding prognosis. We surveyed physicians, nurses, nurse practitioners (NPs), and house staff at the Johns Hopkins Hospital who admit to or practice in one of three surgical ICUs: a 15-bed cardiac surgical ICU that predominantly admits patients after cardiac surgery; a 13-bed surgical ICU and intermediate care unit (IMC) that predominantly admits patients after trauma, transplant, and vascular surgeries; and a 16-bed general surgical ICU and IMC that predominantly admits patients after thoracic, general abdominal, plastic, gynecologic, and ear/nose/throat surgeries. Each surgical ICU operates under a "semi-open" plan; patients are admitted by the primary surgeon with his or her corresponding house-staff team, but with a required ICU team consultation. Thus, decisions are made jointly between the primary surgical and the ICU team. Attending surgeons are board certified or board eligible in surgery. The ICU attending team is interdisciplinary and comprises predominantly physicians with primary board certification in either surgery or anesthesia (a few have primary board certification in medicine or emergency medicine); the majority also concurrently have board certification or are board eligible in critical care. The ICU team comprises either house staff or nurse practitioners, with nurse practitioners having responsibilities similar to those of senior house staff.
Participation in the survey was voluntary. The Johns Hopkins University School of Medicine Institutional Review Board (IRB) approved this research and waived the need for informed consent.

\section{Survey development}

To identify domains for the survey questionnaire, we conducted a literature review and convened focus groups of physicians (surgeons and intensivists) and nurses (ICU staff nurses and NPs) at our academic medical center. The literature review was conducted in PubMed by exploding the terms "communication" and "intensive care unit" and reviewing relevant hits as well as by handsearching personal files. Focus groups were small (two to three individuals), voluntary, and convened with a study investigator (RA) as moderator; comprising nurses, ICU attendings, ICU nurse practitioners, and surgeons who expressed an interest in communication in the ICU, groups were informally asked to list important factors in assessing whether communication between health care providers, and between health care providers and patients, is adequate. The research team reduced and refined the list to include three domains: satisfaction with communication, frequency of communication, and valuation of communication. Within these domains, specific questions called for categoric responses, by using either a nominal ("yes/no") or adjectival Likert scale ("multiple times/day, daily, sometimes, rarely, never" or "always, daily, sometimes, rarely, never"). We pilot tested this questionnaire with a sample of six clinicians, including ICU and surgery physicians and ICU nurses. Based on feedback, the final survey was revised to contain nine questions (Figure 1). At the beginning of our survey, the following definition was given: "For this survey, 'prognosis' is specifically defined as how a patient's illness and overall health is likely to evolve during this hospitalization or over the next few days to months."

\section{Study sample and method of survey administration}

The study sample included full-time ICU nurses, ICU intensivists, or NPs who work in any of the previously described three ICUs; it also included surgeons who admit to any of these three ICUs. All care providers were contacted via an e-mail containing a link to the survey Web page. Potential survey participants were identified by rosters listing full-time employed surgical ICU nurses, intensive care unit attendings or fellows, intensive care unit advanced nurse practitioners, surgeons, surgery house staff, and anesthesia house staff. The survey was facilitated by the Web-based service, SurveyMonkey [24].

\section{Survey statistics and sample size}

Surveys were pooled by group-surgeons, ICU nurses, and ICU physicians and NPs-with the outcomes listed 
1) Demographic question (e.g. - "What is your ICU affiliation?" or "What is your training rank?")

2) Are you generally satisfied with the communication about patient prognosis between the patient/patient family and: The surgical team? The ICU nursing team? The ICU doctor and nurse practitioner team?

3) How often do you speak about your patient's prognosis to the: Patient or patient's family members? ICU nurse? Surgery team? ICU doctor and nurse practitioner team? Social worker?

4) How often does the patient or patient family ask you questions about patient prognosis?

5) Do you feel that you have adequate time to answer a family's questions about patient prognosis?

6) Do you talk to family members about patient prognosis using: The telephone? Email?

7) How often do your patient/patient family members have questions about patient prognosis that you: Cannot answer? Fell you should not answer? Feel uncomfortable answering?

8) If you have concerns about your patient's prognosis, do you feel that you have opportunities to communicate them to the: Surgery team? ICU nurses? ICU doctor and nurse practitioner team? Social worker?

9) If you have concerns about your patient's prognosis, do you feel that they are valued by the: Surgery team? ICU nurses? ICU doctor and nurse practitioner team? Social worker?

as proportions. As the NP role is similar to that of senior ICU house staff, NP responses were listed with the ICU physician group, as opposed to the ICU nurse group. Descriptive statistics were used. Stata software (version 10.1; College Station, TX) was used for all analyses.

\section{Results}

We received 103 responses from a pool of 258 individuals (overall response rate, 40\%). Subclassification by group included a total of 40 responses from surgical ICU nurses (47\% response rate), 39 responses from ICU physicians (52\%), four responses from nurse practitioners (NPs; $50 \%$ response rate), and 20 responses from surgeons (22\% response rate). Further demographics of survey respondents are depicted in Table 1.

Satisfaction with communication regarding prognosis Among respondents who were surgeons, the vast majority were satisfied with their own communication and the communication by ICU physicians, NPs, and ICU staff nurses (Table 2). Three fourths of ICU physicians and NPs were satisfied with their own communication about prognosis; nearly nine of ten were satisfied with that of ICU nurses; and just less than one fourth were satisfied with surgeon communication regarding prognosis. Of ICU nurses, many were satisfied with their own communication and ICU physician and NP communication, and few (3\%) were satisfied with surgeon communication regarding prognosis.

Perceptions of frequency of discussions with families Three fourths of ICU nurses report speaking to patients and patient families daily or multiple times per day regarding prognosis. Forty percent of surgeons and one third of ICU physicians and NPs report speaking to patients and patient families daily or multiple times per day regarding prognosis.

\section{Perceptions of frequency of discussions with other} health care providers

The different types of health care providers varied in how often they reported speaking to each other regarding a patient's prognosis (Table 3). Just less than half of ICU nurses noted that they and surgeons speak to each other 
Table 1 Demographics of survey respondents

\begin{tabular}{|c|c|c|c|c|c|}
\hline $\begin{array}{l}\text { Surgeons } \\
(n=20)\end{array}$ & & ICU physicians and & NPs $(n=41)$ & $\begin{array}{l}\text { ICU staff nurses } \\
(n=40)\end{array}$ & \\
\hline Rank & Number (\%) & Rank & Number (\%) & SICU affiliation & Number (\%) \\
\hline Attending surgeon & $6(30)$ & Attending intensivist & $17(41.5)$ & Cardiac SICU & $15(37.5)$ \\
\hline Surgeon and ICU attending & $1(5)$ & Nurse practitioner & $4(9.8)$ & Trauma, transplant, vascular SICU & $8(20)$ \\
\hline Fellow & $2(10)$ & Fellow & $1(2.4)$ & General SICU & $17(42.5)$ \\
\hline Chief of resident service or senior resident & $7(35)$ & Resident & $19(46.3)$ & & \\
\hline Junior resident or intern & $4(20)$ & & & & \\
\hline
\end{tabular}

ICU, intensive care unit; NP, nurse practitioners; SICU, surgical intensive care unit.

about a patient's prognosis daily or multiple times per day, whereas $60 \%$ of surgeons noted the same conversations occurring. Among ICU physicians and NPs and surgeons, half of ICU physicians and NPs noted that they speak to the surgeon about a patient's prognosis on a daily or multiple times per day basis, whereas a higher percentage of surgeons noted the same conversations occurring. ICU physicians and NPs and ICU nurses noted similar occurrences of speaking to each other daily or multiple times per day regarding a patient's prognosis.

\section{Opportunities for and valuation of discussions}

Ninety percent of surgeons responded that they had multiple opportunities per day to speak with both ICU physicians and NPs and ICU nurses regarding patient prognosis (Table 4). The majority of surgeons also reported that their concerns regarding prognosis were valued by both ICU physicians and NPs and ICU nurses. ICU nurses noted many opportunities to communicate with ICU physicians and NPs, although many thought that their comments were moderately valued by ICU physicians and NPs. ICU nurses noted fewer opportunities to communicate to surgeons and, again, that their concerns were not as valued by surgeons. ICU physicians and NPs noted many opportunities to communicate with ICU nurses and that their concerns were valued by the nurses. However, ICU physicians and NPs had fewer opportunities to communicate with surgeons, and they thought that their comments were less valued.

\section{Discussion}

ICU nurses, surgeons, and ICU intensivists and nurse practitioners varied widely in their satisfaction with

Table 2 Health care provider satisfaction with communication regarding prognosis

\begin{tabular}{lccc}
\hline & \multicolumn{3}{c}{ Person Reviewed } \\
\cline { 2 - 4 } Reviewer & Surgeon & ICU MD/NP & ICU RN \\
\hline Surgeon & $90 \%$ & $85 \%$ & $85 \%$ \\
ICU MD/NP & $23 \%$ & $74 \%$ & $88 \%$ \\
ICU RN & $3 \%$ & $71 \%$ & $82 \%$ \\
\hline
\end{tabular}

$\mathrm{ICU}$, intensive care unit; MD, physician; NP, nurse practitioner; RN, nurse. communication relating to prognosis. Moreover, health care provider groups also varied in whether they thought that they had opportunities to communicate prognosis and whether their concerns were valued by the other providers. In total, these results hint at the nuanced and complicated relationships present in ICUs, particularly surgical ICUs, where nurses, surgeons, and ICU physicians and NPs must work together to provide the care for any single patient.

Previous studies quantify and qualify varying physician and nurse perceptions regarding prognosis and end-oflife care $[25,26]$ as well as illustrate that optimal collaboration between physicians and nurses decreases clinician moral distress $[27,28]$, prevents clinician burnout [29], and improves patient outcomes [30,31]. Moreover, multiple factors, including the educational status of the patient's family, the racial background of the physician, physician uncertainty regarding the patient's prognosis, and conflicts between the patient family and the physician, affect whether physicians discuss prognosis and the language used in that discussion $[5,6,32,33]$. Finally, not all patient families wish to discuss prognosis [34,35] and health care providers should consider using the "asktell-ask" protocol to elicit family member preferences regarding discussions of prognosis [36,37].

Among ICU nurses as well as ICU physicians and NPs, general dissatisfaction existed with how surgeons communicate prognosis, the opportunities to communicate with surgeons regarding prognosis, and whether the conversations were valued by the surgeon. Yet, surgeons reported good communication between themselves and nurses and ICU physicians and NPs. A number of potential causes could be contributing to this. First, the

Table 3 Frequency of discussions concerning prognosisa

\begin{tabular}{lccc}
\hline & \multicolumn{3}{c}{ Recipient } \\
\cline { 2 - 4 } Communicator & Surgeon & ICU MD/NP & ICU RN \\
\hline Surgeon & - & 80 & 60 \\
ICU MD/NP & 53 & - & 81 \\
ICU RN & 43 & 85 & - \\
\hline
\end{tabular}

"Percentage noting "daily or multiple times a day" discussions. ICU, intensive care unit; MD, physician; NP, nurse practitioner; $\mathrm{RN}$, nurse. 
Table 4 Opportunities between health providers to communicate regarding patient prognosis and whether that communication is valued

\begin{tabular}{|c|c|c|c|c|c|c|}
\hline \multirow{3}{*}{ Communicator } & \multicolumn{6}{|c|}{ Recipient } \\
\hline & \multicolumn{2}{|c|}{ Surgeon } & \multicolumn{2}{|c|}{ ICU MD/NP } & \multicolumn{2}{|c|}{ ICU RN } \\
\hline & $\begin{array}{l}\text { Opportunity to } \\
\text { communicate }\end{array}$ & $\begin{array}{c}\text { Communication } \\
\text { valued }\end{array}$ & $\begin{array}{l}\text { Opportunity to } \\
\text { communicate }\end{array}$ & $\begin{array}{c}\text { Communication } \\
\text { valued }\end{array}$ & $\begin{array}{l}\text { Opportunity to } \\
\text { communicate }\end{array}$ & $\begin{array}{c}\text { Communication } \\
\text { valued }\end{array}$ \\
\hline Surgeon & - & - & $90 \%$ & $75 \%$ & $90 \%$ & $90 \%$ \\
\hline ICU MD/NP & $46 \%$ & $27 \%$ & - & - & $85 \%$ & $83 \%$ \\
\hline ICU RN & $29 \%$ & $31 \%$ & $91 \%$ & $63 \$$ & - & - \\
\hline
\end{tabular}

The percentages given indicate "always" or "often." ICU, intensive care unit; MD, physician; NP, nurse practitioner; RN, nurse.

surgeons who responded to the survey could be already interested in communication and thus have better communication skills than their colleagues. Furthermore, it is unclear whether the dissatisfaction is from a lack of communication regarding prognosis or whether the content of the discussion was not thought appropriate (perhaps either overly optimistic or overly pessimistic). ICU physicians, NPs, and nurses may not be aware of discussions surgeons have with patients. Finally, surgeon perceptions of the need for discussing prognosis and comfort with such discussions may also differ from that of ICU nurses, NPs, and physicians.

The advent of ICU daily-goal sheets [38] ensures that communication between the ICU physician and NP team and the ICU nursing team is both timely and follows a protocol. However, goal sheets rarely exist to prompt similar detailed conversations between surgeons and ICU nurses and/or surgeons and ICU physicians and NPs; the miscommunication or lack of communication between surgeons and ICU providers may be a consequence of this lack of a daily goal sheet.

Further cultural reasons may underlie these discrepancies. For example, the concept of "prognosis" may differ between health care provider groups. In her book, Life and Death in Intensive Care [20], Joan Cassell notes that, particularly for patients in surgical ICUs, surgeons, ICU physicians, and nurses often have different "moral economies." In Dr. Cassell's description, surgeons note the enemy to be death, and emphasis is subsequently placed on caring for the physiologic patient with a goal of averting death at all costs. In contrast, ICU physicians and nurses envision the greatest enemy to be suffering, and emphasis is toward averting suffering, particularly if a patient is dying and the suffering is seen as "needless." These different moral economies may derive from the increased time that nurses and ICU teams, as compared with surgeons, spend with suffering ICU patients.

Although Cassell's book and similar articles by Buchman et al. [18] and Penkoske and Buchman [19] address endof-life care, similar principles may also hold true for discussions regarding prognosis. When asked about "communication regarding prognosis," surgeons could be interpreting "prognosis" as being whether the patient can be kept alive for the next day, or until discharge from the surgical ICU or hospital. In contrast, ICU nurses and ICU physicians and NPs could be interpreting "prognosis" to mean whether the patient is likely to have a long hospitalization, a complicated post-hospitalization course of rehabilitation, or the ability for the patient to have a quality of life that is consistent with his or her values and beliefs. Despite having defined "prognosis" in the survey, the term could have been differently interpreted.

Cultural environments-such as differences between surgical, nursing, and ICU physician/NP cultures-can also simplify, or complicate, discussions regarding prognosis. Besides an emphasis on preventing death, aspects of surgical culture that could complicate the communication of prognosis include: the "rescue credo" [17]-the need to "save" or "rescue" a patient from dying; that surgeons often associate patient death with personal failure and shame $[17,20]$ and professional "shame" if a patient does not receive all possible interventions [18].

Less is found in the literature concerning the culture of ICU physicians and NPs, particularly in surgical ICUs. ICU physicians, some of whom are surgeons, and NPs, can have varying opinions on whether the goal of surgical ICU care is to avert death, to avert suffering, or some balance of the two. Moreover, individual opinions within caregiver groups likely vary widely (for example, not all surgeons or nurses have the same views regarding prognosis), and little is known regarding the relative size of within-group versus between-group variation. Moreover, ICU physicians often rotate weekly, leaving little time to bond with families and to discuss prognosis [20]. Nevertheless, little empiric evidence exists that this ICU-physician schedule poses barriers to discussing prognosis.

The ICU nurse has a pivotal role for ICU patients and their families. From a practical standpoint, nurses spend the most time with ICU patients and family members [39], and thus are a valuable resource for identifying what the patient and family understand and whether communication with them is needed or effective. Moreover, nurses often informally provide families with information about prognosis and act as the medical "translator" [20] for 
families, as well as the "power broker" between health care providers, particularly if surgeon and ICU physician and NP teams disagree. Despite this, ICU nurses are often excluded from formal discussions regarding prognosis, limiting their ability to inform these discussions. Such situations can contribute to the all-too-common phenomenon of a patient or patient family hearing contrasting prognoses from varying health care providers.

Multiple limitations to this study exist. First, our sample size was small, and we studied staff from surgical ICUs in one academic medical center; our findings may not be representative of the views of clinicians working in other settings. Second, given the response rates, we cannot exclude the possibility of significant response and nonresponse bias in the survey results. Third, although we provided a working definition of prognosis for purposes of the survey, clinicians may have understood the term differently based on their prior experience. Fourth, our survey instrument was not previously validated; however, to our knowledge, no existing validated tools address these specific issues, and our methods for survey development supported the validity of the instrument we used. Finally, the study measures perceptions and estimations of communication, which is potentially unreliable. However, studies measuring actual quality and content of this communication are cumbersome, and, as perceptions and knowledge drive actions, we consider it worthwhile to assess those perceptions.

The results of our study highlight potential targets for ICU-performance improvement initiatives. Daily goal sheets for surgeons and ICU teams (nurses and/or ICU physicians and NPs) could direct the content of conversations as well as facilitate more frequent opportunities for discussions. Moreover, routine multidisciplinary family meetings for ICU patients and patient families could further mitigate deficiencies highlighted by the surveys. Finally, as palliative care teams become more prominent in the ICU, or as surgeons, ICU physicians and NPs, and ICU nurses become more skilled in palliative care, such discussions regarding prognosis may also become less problematic.

Although optimal communication in the ICU is difficult, it is especially difficult in surgical ICUs where patients are often cared for by two sets of physicians. This study highlights the need for further additional research in surgical ICU communication, particularly exploring patient and patient family opinions, and for focused efforts to improve communication.

\section{Conclusions}

ICU nurses, surgeons, and ICU intensivists and NPs varied widely in their satisfaction with communication relating to prognosis. These same clinician groups also varied in whether they thought that they had opportunities to communicate prognosis and whether their concerns were valued by other clinicians. These variations could result from practical circumstances (some groups may be unaware of discussions that do occur); system failures (tools that facilitate discussions, such as daily goal sheets, may not exist); or cultural differences (different groups have varying expectations about when communication should occur and what should be the content of that communication). Further research, particularly into the communication expected and desired by surgical ICU patients and their families, is needed.

\section{Key messages}

- Optimal care for surgical ICU patients requires clear communication between ICU nurses, surgeons, and ICU physicians and mid-level providers.

- Not all health care provider teams in surgical ICUs are satisfied with how other teams communicate prognosis to patients.

- ICU nurses are the most likely to report speaking at least daily to patients and patient families about prognosis.

- Some health care provider teams, particularly ICU nurses, report often not having opportunities to communicate about patient prognosis, and that when communication does occur, their input is less valued by other providers.

- Varying levels of health care provider satisfaction in communication regarding prognosis may be a result of practical barriers, such as a lack of daily goal sheets spurring communication between surgeon and ICU teams, as well as cultural barriers, such as whether a "bad prognosis" specifically refers to imminent patient death or may instead refer to patient suffering, such as the inability for a patient to recover a quality of life that is consistent with his or her values and beliefs.

\section{Abbreviations}

ICU: intensive care unit; IMC: intermediate care unit; MD: physician; NP: nurse practitioner; RN: registered nurse; SICU: surgical intensive care unit.

\section{Acknowledgements}

RA was salary-supported by a T32 National Institute of Health grant throughout this study.

\section{Author details}

'Department of Anesthesiology and Critical Care Medicine, The Johns Hopkins School of Medicine, 600 North Wolfe Street, Baltimore, MD, 21287, USA. ${ }^{2}$ Department of Surgical Nursing, The Johns Hopkins Hospital, 600 North Wolfe Street, Baltimore, MD, 21287, USA. ${ }^{3}$ Department of Surgery and Oncology, The Johns Hopkins University School of Medicine, 600 North Wolfe Street, Baltimore, MD, 21287, USA. ${ }^{4}$ Department of Medicine, Division of Pulmonary, Critical Care and Sleep Medicine and Hertzberg Palliative Care Institute, Mount Sinai School of Medicine, One Gustave L. Levy Place, New York, NY, 10029, USA. 


\section{Authors' contributions}

RA conceived and designed the studies, wrote and distributed the surveys, analyzed the data, and drafted the manuscript. RW distributed surveys, analyzed data, and was influential in drafting the manuscript. DS distributed surveys. MZ distributed surveys and analyzed data. NA distributed surveys, provided idea content for the discussion section of the manuscript, and was influential in the drafting of the manuscript. JN aided in conception of the study and provided idea content for the discussion section of the manuscript. PP aided in conceiving and designing the study, analyzed the data, and provided idea content for the discussion section of the manuscript. All authors read and approved the final manuscript.

\section{Competing interests}

The authors declare that they have no competing interests.

Received: 28 June 2010 Revised: 13 September 2010

Accepted: 29 November 2010 Published: 29 November 2010

\section{References}

1. Hanson LC, Danis M, Garrett J: What is wrong with end-of-life care? opinions of bereaved family members. J Am Geriatr Soc 1997, 45:1339-1344.

2. Heyland DK, Dodek P, Rocker G, Groll D, Gafni A, Pichora D, Shortt S, Tranmer J, Lazar N, Kutsogiannis J, Lam M, Canadian Researchers End-of-Life Network (CARENET): What matters most in end-of-life care: perceptions of seriously ill patients and their family members. CMAJ 2006, 174:627-633.

3. Nelson JE, Puntillo KA, Pronovost PJ, Walker AS, MCAdam JL, llaoa D, Penrod JD: In their own words: patients and families define high-quality palliative care in the intensive care unit. Crit Care Med 2010, 38:808-818

4. Apatira L, Boyd EA, Malvar G, Evans LR, Luce JM, Lo B, White DB: Hope, truth, and preparing for death: perspectives of surrogate decision makers. Ann Intern Med 2008, 149:861-868.

5. Evans LR, Boyd EA, Malvar G, Apatira L, Luce JM, Lo B, White DB: Surrogate decision-makers' perspectives on discussing prognosis in the face of uncertainty. Am J Respir Crit Care Med 2009, 179:48-53.

6. Boyd EA, Lo B, Evans LR, Malvar G, Apatira L, Luce JM, White DB: "It's not just what the doctor tells me": factors that influence surrogate decisionmakers' perceptions of prognosis. Crit Care Med 2010, 38:1270-1275.

7. Schneiderman LJ, Gilmer T, Teetzel HD, Dugan DO, Blustein J, Cranford R, Briggs KB, Komatsu Gl, Goodman-Crews P, Cohn F, Young EW: Effect of ethics consultations on nonbeneficial life-sustaining treatments in the intensive care setting: a randomized controlled trial. JAMA 2003, 290:1166-1172.

8. Campbell ML, Guzman JA: Impact of a proactive approach to improve end-of-life care in a medical ICU. Chest 2003, 123:266-271.

9. Lilly CM, De Meo DL, Sonna LA, Haley KJ, Massaro AF, Wallace RF, Cody S: An intensive communication intervention for the critically ill. Am J Med 2000, 109:469-475

10. Mosenthal AC, Murphy PA, Barker LK, Lavery R, Retano A, Livingston DH: Changing the culture around end-of-life care in the trauma intensive care unit. J Trauma 2008, 64:1587-1593.

11. Truog RD, Campbell ML, Curtis JR, Haas CE, Luce JM, Rubenfeld GD, Rushton CH, Kaufman DC, American Academy of Critical Care Medicine: Recommendations for end-of-life care in the intensive care unit: a consensus statement by the American College [corrected] of Critical Care Medicine. Crit Care Med 2008, 36:953-963.

12. Mularski RA, Curtis JR, Billings JA, Burt R, Byock I, Fuhrman C, Mosenthal AC, Medina J, Ray DE, Rubenfeld GD, Schneiderman LJ, Treece PD, Truog RD, Levy MM: Proposed quality measures for palliative care in the critically ill: a consensus from the Robert Wood Johnson Foundation Critical Care Workgroup. Crit Care Med 2006, 34(11 suppl):S404-S411.

13. Clarke EB, Curtis JR, Luce JM, Levy M, Danis M, Nelson J, Solomon MZ, Robert Wood Johnson Foundation Critical Care End-Of-Life Peer Workgroup Members: Quality indicators for end-of-life care in the intensive care unit. Crit Care Med 2003, 31:2255-2262.

14. Davidson JE, Powers K, Hedayat KM, Tieszen M, Kon AA, Shepard E, Spuhler V, Todres ID, Levy M, Barr J, Ghandi R, Hirsch G, Armstrong D, American College of Critical Care Medicine Task Force 2004-2005, Society of
Critical Care Medicine: Clinical practice guidelines for support of the family in the patient-centered intensive care unit: American College of Critical Care Medicine Task Force 2004-2005. Crit Care Med 2007, 35:605-622.

15. Lanken PN, Terry PB, Delisser HM, Fahy BF, Hansen-Flaschen J, Heffner JE, Levy M, Mularski RA, Osborne ML, Prendergast TJ, Rocker G, Sibbald WJ, Wilfond B, Yankaskas JR, ATS End-of-Life Care Task Force: An official American Thoracic Society clinical policy statement: palliative care for patients with respiratory diseases and critical illnesses. Am J Respir Crit Care Med 2008, 177:912-927.

16. Easson AM, Crosby JA, Librach SL: Discussion of death and dying in surgical textbooks. Am J Surg 2001, 182:34-39.

17. Bosk CL: Forgive and Remember: Managing Medical Failure Chicago: University of Chicago Press; 1979.

18. Buchman TG, Cassell J, Ray SE, Wax ML: Who should manage the dying patient? Rescue, shame, and the surgical ICU dilemma. J Am Coll Surg 2002, 194:665-673.

19. Penkoske PA, Buchman TG: The relationship between the surgeon and the intensivist in the surgical intensive care unit. Surg Clin North Am 2006, 86:1351-1357.

20. Cassell J: Life and Death in Intensive Care Philadelphia: Temple University Press; 2005.

21. Wlody GS: Nursing management and organizational ethics in the intensive care unit. Crit Care Med 2007, 35(2 suppl):S29-S35.

22. Pendry PS: Moral distress: recognizing it to retain nurses. Nurs Econ 2007, 25:217-221.

23. Lipsett PA, Swoboda SM, Dickerson J, Ylitalo M, Gordon T, Breslow M, Campbell K, Dorman T, Pronovost P, Rosenfeld B: Survival and functional outcome after prolonged intensive care unit stay. Ann Surg 2000, 231:262-268

24. SurveyMonkey. [http://www.surveymonkey.com].

25. Ferrand E, Lemaire F, Regnier B, Kuteifan K, Badet M, Asfar P, Jaber S, Chagnon JL, Renault A, Robert R, Pochard F, Herve C, Brun-Buisson C, Duvaldestin P, French RESSENTI Group: Discrepancies between perceptions by physicians and nursing staff of intensive care unit endof-life decisions. Am J Respir Crit Care Med 2003, 167:1310-1315.

26. Westphal DM, McKee SA: End-of-life decision making in the intensive care unit: physician and nurse perspectives. Am J Med Qual 2009, 24:222-228.

27. Hamric AB, Blackhall LJ: Nurse-physician perspectives on the care of dying patients in intensive care units: collaboration, moral distress, and ethical climate. Crit Care Med 2007, 35:422-429.

28. Schmalenberg C, Kramer M: Nurse-physician relationships in hospitals: 20,000 nurses tell their story. Crit Care Nurse 2009, 29:74-83.

29. Danjoux Meth N, Lawless B, Hawryluck L: Conflicts in the ICU: perspectives of administrators and clinicians. Intensive Care Med 2009, 35:2068-2077.

30. Baggs JG, Ryan SA, Phelps CE, Richeson JF, Johnson JE: The association between interdisciplinary collaboration and patient outcomes in a medical intensive care unit. Heart Lung 1992, 21:18-24.

31. Baggs JG, Schmitt MH, Mushlin Al, Mitchell PH, Eldredge DH, Oakes D, Hutson AD: Association between nurse-physician collaboration and patient outcomes in three intensive care units. Crit Care Med 1999, 27:1991-1998

32. White DB, Engelberg RA, Wenrich MD, Lo B, Curtis JR: The language of prognostication in intensive care units. Med Decis Making 2010, 30:76-83.

33. White DB, Engelberg RA, Wenrich MD, Lo B, Curtis JR: Prognostication during physician family discussions about limiting life support in intensive care units. Crit Care Med 2007, 35:442-448.

34. Carrese JA, Rhodes LA: Western bioethics on the Navajo reservation: benefit or harm? JAMA 1995, 274:826-829.

35. Schaefer KG, Block SD: Physician communication with families in the ICU: evidence-based strategies for improvement. Curr Opin Crit Care 2009, 15:569-577.

36. Back AL, Arnold RM, Baile WF, Tulsky JA, Fryer-Edwards K: Approaching difficult communication tasks in oncology. CA Cancer J Clin 2005, 55:164-177.

37. Buckman R: How to Break Bad News: a Guide for Health Care Professionals Baltimore: Johns Hopkins University Press; 1992. 
38. Winters B, Dorman T: Patient-safety and quality initiatives in the intensive-care unit. Curr Opin Anaesthesiol 2006, 19:140-145.

39. Asch DA, Shea JA, Jedrziewski MK, Bosk CL: The limits of suffering: critical care nurses' views of hospital care at the end of life. Soc Sci Med 1997, 45:1661-1668

\section{doi:10.1186/cc9346}

Cite this article as: Aslakson et al:: Surgical intensive care unit clinician estimates of the adequacy of communication regarding patient prognosis. Critical Care 2010 14:R218.

Submit your next manuscript to BioMed Central and take full advantage of:

- Convenient online submission

- Thorough peer review

- No space constraints or color figure charges

- Immediate publication on acceptance

- Inclusion in PubMed, CAS, Scopus and Google Scholar

- Research which is freely available for redistribution

Submit your manuscript at www.biomedcentral.com/submit 